Do the severity of Intellectual Disability and /or the presence of neurodevelopmental disorders influence the onset of dementia in people with Down syndrome?

\title{
Anderson, $\mathrm{M}$
}

http://hdl.handle.net/10026.1/17177

10.1080/19315864.2020.1822964

Journal of Mental Health Research in Intellectual Disabilities

Informa UK Limited

All content in PEARL is protected by copyright law. Author manuscripts are made available in accordance with publisher policies. Please cite only the published version using the details provided on the item record or document. In the absence of an open licence (e.g. Creative Commons), permissions for further reuse of content should be sought from the publisher or author. 
See discussions, stats, and author profiles for this publication at: https://www.researchgate.net/publication/344934500

Do the severity of Intellectual Disability and /or the presence of neurodevelopmental disorders influence the onset of dementia in people with Down syndrome?

Article in Journal of Mental Health Research in Intellectual Disabilities · October 2020 DOI: $10.1080 / 19315864.2020 .1822964$

CITATIONS

0

5 authors, including:

Merryn Anderson

National Health Service

1 PUBLICATION OCITATIONS

SEE PROFILE

Karen Dodd

Surrey and Borders Partnership NHS Foundation Trust

32 PUBLICATIONS 305 CITATIONS

SEE PROFILE

Some of the authors of this publication are also working on these related projects:

psychological skills View project

SUDEP Safety - person centred communication of risk View project
READS

42

Katy Oak

Royal Cornwall Hospitals NHS Trust

9 PUBLICATIONS 33 CITATIONS

SEE PROFILE

Rohit Shankar

University of Plymouth

191 PUBLICATIONS 944 CITATIONS

SEE PROFILE 
Do the severity of Intellectual Disability and /or the presence of neurodevelopmental disorders influence the onset of dementia in people with Down syndrome?

Key Words

Down syndrome; Intellectual Disability; Dementia; Neurodevelopmental disorder 


\begin{abstract}
Introduction

Having a diagnosis of Down syndrome (DS) is associated with intellectual disability (ID), pervasive developmental disorders and Alzheimer's dementia (AD). The association between these conditions has not been well evaluated. This paper looks to examine the current evidence pertaining to the relationship between dementia in people with DS and severity of ID and the presence of pervasive developmental disorders.
\end{abstract}

\title{
Methods
}

A scoping review using PRISMA guidance was undertaken. Medline, Cochrane database, NHS evidence, Trials registers and Open Grey were searched in December 2018 and an updated search was completed in July 2020. Three search strategies were used to retrieve articles relating to DS, dementia, pervasive developmental disorders (including autism, autism spectrum disorder (ASD) and attention deficit hyperactivity disorder (ADHD)) and severity of ID. Studies were included if they met the pre-defined inclusion criteria of investigating an association between autism/ASD, ADHD, or severity of ID and the development of dementia in people with DS. Studies were excluded if they did not include primary data, if the population included non-Down causes of ID, or if no specific outcome measure related to comorbid autism/ASD, ADHD, or severity of ID and dementia in people with a diagnosis of DS were reported. There were no exclusions related to study design. Papers were assessed for quality using the Mixed Methods Appraisal Tool (MMAT; Hong et al., 2018).

\section{Results}

The search identified fifteen papers, publishing results from twelve studies, relating to severity of ID, DS and dementia. No papers were identified relating to pervasive developmental disorders, DS and dementia. There is limited evidence on how severity of ID impacts on the presentation, diagnosis, management or prognosis of dementia in people with DS. However, no evidence was found on comorbid pervasive developmental disorders, DS and dementia.

\section{Conclusion}

This paper has identified multiple areas for future research. There is an urgent need for longitudinal studies into the presentation, development and progression of dementia in people with DS ensuring the severity of ID and comorbid pervasive developmental conditions are captured regularly to understand their influence on the dementia aetiology and outcome. 


\section{Introduction}

The term Down Syndrome (DS) refers to a condition that is associated with intellectual disability (ID), having a small head, ears and mouth, upward slanting eyes and a flattened facial profile and nose (NICHD, 2017a). It is caused by the affected person having trisomy 21 (Lejeune et al., 1959). In 2013 it was estimated that there was a live birth rate of people with DS of 1.0 per 1,000 live births in the United Kingdom (UK; Morris \& Springett, 2014). A paper looking at the United States population estimated a prevalence of people with DS of 8.27 per 10000 people (Presson et al., 2013).

Large numbers of people with DS encounter various additional health issues (Antonarakis et al., 2004, Leonard \& Wen, 2002). Specifically physical health comorbidities including: congenital heart defects, congenital gastrointestinal abnormalities, vision and hearing problems, susceptibility to infections, and endocrine abnormalities particularly hypothyroidism (NICHD, 2017b). People with DS are also commonly affected by conditions that affect their cognitive profile. This includes neurodevelopmental conditions that are present from childhood and dementia that causes a progressive decline in cognition in adulthood (NICHD, 2017a). Specific conditions that are frequently diagnosed in people with DS and affect baseline cognitive profile include intellectual disability (ID) and neuro-developmental and pervasive developmental disorders such as autism, autism spectrum disorders (ASD) and attention deficit hyperactivity disorder (ADHD; Ekstein et al., 2011, Moss et al., 2013, Richards et al., 2015, Walsh et al., 2015, Yahia et al., 2014).

\section{Association of DS and dementia}

DS is particularly associated with an increased risk of Alzheimer's dementia (AD) due to overexpression of genes found on chromosome 21 including the amyloid precursor protein (Wiseman et al., 2018). The proportion of people with DS who develop dementia is estimated to increase from 0-33\% in those aged 30-39, to $15-77 \%$ in those aged over 60 years (Head et al, 2012). The British Psychological Society and the Royal College of Psychiatrists have published guidance on management of people with ID who develop dementia. They highlight the importance of thorough baseline assessment and proactive monitoring over time to allow accurate and timely diagnosis of dementia (BPS, 2015).

\section{Association of DS and ID}

All people with a diagnosis of DS are recognised to have co-morbid ID, though the severity of ID can vary from mild to profound. A study found that in a cohort of 114 people with DS, $35.1 \%$ had mild ID, $38.6 \%$ had moderate ID, and $26.3 \%$ had severe or profound ID based on standardised intelligence tests (Walsh et al., 2015). People with ID (without DS) have an increased risk of dementia compared to the general population with one study finding a standardised incidence ratio compared to the general population of 4.98 (Strydom et al., 2013).

\section{Association of DS and Autism/ASD}

ASD includes several conditions that used to be diagnosed separately: autistic disorder, pervasive developmental disorder not otherwise specified (PDD-NOS), and Asperger syndrome. These 
conditions are now all called autism spectrum disorder. The predominant disorder in this group is childhood autism. The two, autism and ASD are at times used synonymously. Autism and ASD are frequently co-morbid with a diagnosis of DS, one study found in a population of people with DS (age range 4 to $62, \mu=22.16), 19.4 \%$ met the diagnostic criteria for ASD and $8.3 \%$ for a diagnosis of autism (Moss et al., 2013). In those with ASD the prevalence of comorbid DS is 16\% (Richards et al., 2015). ASD and Autism are associated with impairment in cognitive domains such as attention, working memory, and fluency and this impairment has been found to continue into middle and old age (Geurts \& Vissers, 2012). Adults with ASD or Autism have been shown to perform less well on executive function tests compared to controls. In particular they showed weakness in tasks of assessing flexibility, working memory and inhibition (Braden et al., 2017). The aspects of cognition affected in autism/ASD are also affected in the development of dementia in people with DS, potentially leading to altered presentation and progression and consequent diagnostic uncertainty and difficulty in assessment (Sauna-Aho et al., 2018). There is little longitudinal evidence that investigates how the baseline cognitive profile of someone with ASD/Autism changes as they age and/or develop dementia (Braden et al., 2017).

\section{$\underline{\text { Association of DS and } A D H D}$}

ADHD is commonly diagnosed in people with DS. One study of children with DS (age range $5-16, \mu$ $=8$ ) found a prevalence of ADHD of around 43.9\% (Ekstein et al., 2011). A cross-sectional sibling paired study comparing siblings without DS to their sibling with DS, found ADHD in $8 \%$ of the siblings without DS compared to $56 \%$ of those with a diagnosis of DS, implicating a genetic rather than environmental cause for the increased risk (Yahia et al., 2014). Similarly to ASD, ADHD is associated with cognitive impairment which continues into adulthood (Das et al., 2015). A longitudinal study found that $78 \%$ of children with ADHD showed some evidence of persistence at a 10 year follow up (although this paper was not focussed on ADHD in people with DS; Biederman et al., 2010). In those with adult $A D H D$ in general population studies, cognitive symptoms have been found to overlap with the symptoms of cognitive decline as seen in mild cognitive impairment $(\mathrm{MCl})$ for example impaired attention, impaired organisation and increased forgetfulness (Kooij et al., 2016). Although symptoms of ADHD are present from childhood, it is sometimes not diagnosed until adulthood. In adults it is the cognitive rather than behavioural symptoms of ADHD that predominate creating the potential for misdiagnosis as $\mathrm{MCl}$ (Callahan et al., 2017). As such, without a baseline assessment of a person's ADHD symptoms and cognitive profile, a diagnosis of dementia may be complicated due to altered presentation and progression of symptoms. It is important that a diagnosis of ADHD is not missed or misdiagnosed as early dementia, potentially denying effective treatment (NICE, 2018a).

It is possible that different baseline cognitive profiles caused by these conditions may alter the presentation and progression of dementia in people with DS; understanding this is important to enable accurate and timely diagnosis. The diagnosis of dementia in people with DS relies on the assessment of change in cognition over time,. Without clear baseline assessment of conditions that affect a person's cognitive profile diagnosis of dementia and prognostic evaluation could be wrong. The frequency of co-morbid ID, autism/ASD and ADHD found in people who have DS, and each conditions independent potential contribution to cognitive impairment raises concerns if there is adequate evidence to help clinicians understand their compounding impact. This paper seeks to 
examine the current evidence relating to these conditions in people with DS and the development of dementia.

\section{Aim}

To conduct a scoping review to explore the available evidence pertaining to-

1. The relationship between dementia in people with DS and the severity of ID.

2. The relationship between dementia in people with DS and the presence of neurodevelopmental/pervasive developmental disorders (autism, ASD, ADHD).

\section{Methods}

\section{Data sources}

This paper utilises the Preferred Reporting Items for Systematic reviews and Meta-Analyses extension for Scoping Reviews (PRISMA-SCR) guidance to provide the review structure (PRISMA, 2018) (supplementary information 1). A literature search was conducted using Medline, Cochrane database, NHS evidence, Trials registers and Open Grey in December 2018, a further search in July 2020 was completed to check for more recent papers. Three separate searches were completed. Each search strategy utilised a combination of text words and thesaurus terms. The first, second and third searches aimed to retrieve articles in relation to the following

$1^{\text {st }}$. DS, dementia, and autism, ASD/ pervasive developmental disorder

$2^{\text {nd }}$. DS, dementia, and ADHD

$3^{\text {rd }}$. DS, dementia, and the severity of ID

The search was conducted by a specialist librarian (KO) experienced in systematic review searches. The search terms, permutations and combinations are presented in appendix 1 . No limits were applied.

\section{Study selection}

Titles and abstracts were screened for relevance to establish whether they met the inclusion criteria by two reviewers (MA \& RS). Studies were included if they investigated an association between pervasive developmental disorder, ADHD, or severity of ID and the development of dementia in people with DS i.e. not simply people with more severe ID scoring worse on cognitive testing. Full texts were reviewed for exclusion by two reviewers (MA and RS), disagreements were resolved through consensus. Studies were excluded if they did not include primary data or if results were only available for multiple causes of ID. There were no exclusions related to study design. Although review articles without primary data were excluded from the results they were used to identify additional references.

Papers were assessed for quality using the Mixed Methods Appraisal Tool, this tool allows studies using different methodologies to be compared. For each study type there are 5 specific criteria to allow quality assessment and comparison (MMAT; Hong et al., 2018).

\section{Results}


The three searches identified the following number of papers (Table 1, supplementary information 1).

\section{Please insert table 1 here}

Of these, 15 papers satisfied the full inclusion criteria for the 'ID' search ( $3^{\text {rd }}$ search) and no papers were identified for the 'ADHD' or the 'autism/ASD/pervasive developmental disorder' searches $\left(1^{\text {st }}\right.$ and $2^{\text {nd }}$ searches).

\section{Inset PRISMA diagrams here}

Of the fifteen papers identified, five published results at different time points from two different studies. Cosgrave et al. (2000), McCarron et al. (2014), and McCarron et al. (2017) all published results from a study looking at people with DS from the Daughters of Charity Service. KrinskyMcHale, Devenny \& Silverman (2002) and Krinsky-McHale et al. (2008) published results from a longitudinal study of people with DS. Two papers used a cross-sectional approach but used data obtained from people with DS enrolled in the same longitudinal study (Holland et al., 1998, Ball et al., 2008). For the purpose of this review the results of the most recent papers from the longitudinal studies will be included unless otherwise indicated. The two papers using a cross-sectional approach on the same patient population have been kept separate as the outcome measures for each are different (Holland et al., 1998, Ball et al., 2008).

Studies were conducted in North America (Head et al., 2011, Krinsky-McHale et al., 2008, Temple et al., 2001), and Europe (Ball et al., 2008, Deb, Hare \& Prior, 2007, Hithersay et al., 2019, Holland et al., 1998, Johansson, 2002, Margallo-Lana et al., 2007, McCarron et al., 2017, Oliver et al., 1998, Sinai et al., 2018). One study's participants were all from an institutionalised setting (Margallo-Lana et al., 2007). Details of the studies can be found in table two.

\section{Please insert table 2 here}

Key methodological problems found in the identified studies were small number of participants, opportunistic participant recruitment/ data of a sub-set from a pre-existing study, recall bias, low use of objective and validated outcome measures, short follow up times and limited statistical analysis. The MMAT was used to assess papers for quality (MMAT; Hong et al., 2018). Of the papers identified one had a MMAT score of 2/5 (Johansson, 2002), seven scored 3/5 (Ball et al., 2008, Holland et al., 1998, Krinsky-McHale et al., 2008, Margallo-Lana et al., 2007, McCarron et al., 2017, Oliver et al., 1998, Temple et al., 2001), two scored $4 / 5$ (Head et al., 2011, Hithersay et al., 2019) and two scored 5/5 (Deb, Hare \& Prior, 2007, Sinai et al., 2018).

\section{Study design and assessment of baseline ID}

Of the twelve studies identified six were longitudinal (Hithersay et al., 2019, Krinsky-McHale et al., 2008, Margallo-Lana et al., 2007, McCarron et al., 2017, Oliver et al., 1998, Sinai et al., 2018), four were cross sectional (Ball et al., 2008, Holland et al., 1998, Johansson, 2002, Temple et al., 2001), one was a case-control study (Head et al., 2011) and one was a qualitative study (Deb, Hare \& Prior, 2007). 
The longitudinal studies mainly utilised a programme of repeated cognitive measures allowing individual change in cognition to be assessed over time (Hithersay et al., 2019, Krinsky-McHale et al., 2008, Margallo-Lana et al., 2007, McCarron et al., 2017, Oliver et al., 1998). One study was a retrospective longitudinal study which was able to make use of data from assessments that had been completed as a normal part of care (Sinai et al., 2018). These studies used notes review (Krinsky-McHale et al., 2008, McCarron et al., 2017), carer interview (Hithersay et al., 2019) or patient assessment (Margallo-Lana et al., 2007, Oliver et al., 1998) to assess baseline severity of ID. The four studies that utilised a cross-sectional approach were able to compare assessment data between different individuals (Ball et al., 2008, Holland et al., 1998, Johansson, 2002, Temple et al., 2001). To establish baseline level of ID these papers used either historical data from the patient's records (Ball et al., 2008, Temple et al., 2001), carer interview (Holland et al., 1998, Johansson, 2002) or British Picture Vocabulary Scale (BPVS) II score (Ball et al., 2008). BPVS II score provides an estimate of baseline ID despite presence of cognitive decline. The case-control study compared people with DS, with and without dementia (Head et al., 2011). This paper was able to access a full scale Intelligence Quotient (IQ) assessment for each participant to establish baseline severity of ID. Finally, one paper was a qualitative study which did not publish how baseline ID was assessed but used ICD-10 criteria to differentiate severity levels (Deb, Hare \& Prior, 2007).

\section{Exclusions}

Some of the studies excluded participants if their severity of ID/functioning fell below a certain threshold (Ball et al., 2008, Krinsky-McHale et al., 2008, Oliver et al., 1998). In one paper this threshold was not predefined, but was added in the analysis stage, as the participants were found to have not performed above the floor level on outcome measures therefore limiting assessment of change (Ball et al., 2008).

\section{Outcome measures used to assess cognition/cognitive change}

In order to assess cognitive function, multiple outcome measures were used in the studies with most using an assessment battery to allow different aspects of cognition and functioning to be measured. The purpose of this review was not to assess which assessment batteries were most successful in accurately and reliably diagnosing dementia in this patient group. The cognitive and functional assessments used are described in more detail in Table 2.

Impact on severity of ID on the development of dementia in people with DS

\section{Natural history of dementia in people with DS}

Eight of the studies found looked at the development of dementia in those with varying severities of ID. Three studies reported that severity of ID was not associated with the development of dementia nor could it predict the onset of dementia (Hithersay et al., 2019, Holland et al., 1998, McCarron et al., 2017). One study found frontal lobe dementia was more commonly diagnosed in more severe ID (8.9\% in mild-moderate ID vs $21.1 \%$ in severe-profound ID) and AD was a more common diagnosis on mild-moderate ID (CAMDEX diagnosis of AD in $16.1 \%$ with mild-moderate ID vs $5.3 \%$ in severeprofound ID). However this paper is from 1998 so diagnostic workup could have changed. 
Furthermore it is not clear from the paper as to whether calculations to confirm statistical significance were done (Holland et al, 1998).

One study found severity of ID was associated with the degree of cognitive decline, with those with a greater degree of pre-existing cognitive impairment having less of a decline in cognitive assessment score (Margallo-Lana et al., 2007). Another study found that although performance on initial cognitive assessment differed depending on severity of ID, the rate of change in assessment score did not differ between different ID severities (Krinsky-McHale et al., 2008). However, another study found that the rate of cognitive decline was faster in those who had a greater degree of pre-existing cognitive impairment (Oliver et al., 1998). One study assessing the impact of multiple variables on cognitive decline calculated semi-partial correlations and found that baseline level of cognitive function accounted for $12 \%$ of the variation in decline in combined neuropsychological and Dementia Scale for Down Syndrome (DSDS) scores (Temple et al., 2001).

One prospective longitudinal study that published results at different time points found a significant difference in the duration of dementia depending on the severity of ID in their initial 14 year follow up paper but by the 20 year follow up this difference was no longer found (McCarron et al., 2014, McCarron et al., 2017). However, a retrospective longitudinal study found that severity of ID was a significant predictor of survival (Sinai et al., 2018).

\section{Symptoms of dementia}

The number or nature of dementia symptoms differed depending on severity of baseline ID (Ball et al., 2008, Cosgrave et al., 2000, Deb, Hare \& Prior, 2007, Johansson, 2002). There was inconsistency about the development of memory loss as a symptom depending on severity of ID. One study found no association between reported memory decline and BPVS II score (where BPVS II score is used as an estimate of baseline severity of ID; Ball et al., 2008). Another found that memory loss was the most common first symptom of dementia in people with both moderate and severe ID. However those with severe ID more frequently presented with epilepsy or urinary incontinence as the first symptom of dementia compared to those with moderate ID (this did not reach statistical significance; Cosgrave et al., 2000). This finding was not discussed in subsequent papers published from the same study (McCarron et al., 2014, McCarron et al., 2017). There was one qualitative study which looked at initial symptoms of dementia as recalled by a carer. They found that the initial symptoms were thematically different depending on the severity of ID. Those with mild ID showed more symptoms of general forgetfulness and wandering. Those with moderate ID had more symptoms suggestive of personality change e.g. social withdrawal or disinhibition. Those with severe ID had symptoms of not being able to recognise familiar people (Deb, Hare \& Prior, 2007). Another study noted that those with severe ID often operate on visual, rather than verbal recognition resulting in the participants with more severe ID having increased difficulty verbalising memories, therefore introducing a floor effect on some assessments (Johansson, 2002).

\section{Discussion}

Having DS is associated with conditions that affect baseline cognitive ability for example ID and neuro-developmental disorder (Ekstein et al., 2011, Moss et al., 2013, Richards et al., 2015, Walsh et 
al., 2015, Yahia et al., 2014). People with DS are at increased risk of dementia causing their cognitive profile to change over time. This presents a challenge when diagnosing dementia. Timely and accurate diagnosis of dementia is a priority as it affects pharmacological and non-pharmacological management, future planning and carer support (NICE, 2018b).

This scoping review has shown that there has been continued interest in how severity of ID impacts on the development of dementia in people with DS. However, this has often been a secondary consideration to the impact of severity of ID on cognitive assessment. There is limited evidence regarding how different severities of ID may change the initial symptoms of dementia, how these symptoms progress or the impact of dementia on people's lives.

Strikingly, our review found no research that specifically looked at the influence of autism/ASD/pervasive developmental disorder/ADHD on the development of dementia in people with DS. Most studies looking at ASD / autism / pervasive developmental disorder / ADHD focus on children and young adults. In light of this it is maybe not so surprising that there are no studies looking at these comorbidities and dementia, which occurs later in life.

Unlike dementia these conditions are neurodevelopmental and as such will not cause evolving changes in function in adulthood. However, the co-morbidity and impact on baseline cognitive profile of these diagnoses may alter the presentation and progression of dementia.

The studies identified in this review present inconclusive evidence related to if the severity of ID impacts the development and symptomatology of dementia in people with DS. Conflicting evidence from the identified studies means there is insufficient evidence regarding if severity of ID impacts on the development of dementia or its progression. Results ranged from those with more severe ID experiencing less decline on repeated cognitive assessment (Margallo-Lana et al., 2007), severity of ID not affecting rate of change in assessment score (Krinsky-McHale et al., 2008), to those with more severe ID experiencing a faster rate of cognitive decline (Oliver et al., 1998). There was also conflicting evidence regarding if severity of ID affected the duration of dementia. One prospective longitudinal study that published results at different time points found a significant difference in the duration of dementia depending on the severity of ID, with those with more severe ID having dementia for longer, in their initial 14 year follow up paper but by the 20 year follow up this difference was no longer found (McCarron et al., 2014, McCarron et al., 2017). However, a retrospective longitudinal study found that severity of ID was a significant predictor of survival, with those with more severe ID having a lower survival time from dementia diagnosis (Sinai et al., 2018).

There were some differences in the number and nature of dementia symptoms in people with DS depending on the severity of ID (Ball et al., 2008, Cosgrave et al., 2000, Deb, Hare \& Prior, 2007, Johansson, 2002). As would be expected from the diagnostic criteria for dementia, memory loss was an important symptom of dementia regardless of severity of ID (Ball et al., 2008, Cosgrave et al., 2000). However, reported symptoms of dementia did vary depending on severity of ID indicating the need for carer perspectives to be included as part of the diagnostic process (Cosgrave et al., 2000, Deb, Hare \& Prior, 2007). It is important to note that there may also be a difference between which symptoms are noticed first compared to which changes occur first. This would benefit from clarification in future studies. Severity of ID affected how people with DS performed on different cognitive assessments in particular those with more severe ID relied on visual, rather than verbal 
recognition (Johansson, 2002). This indicated the need for cognitive assessment to be tailored to account for the baseline severity of ID.

People with DS and more severe ID were excluded from some of the papers found. This was largely reported to be due to floor effects limiting the ability of cognitive assessments to detect cognitive decline (Ball et al., 2008, Krinsky-McHale et al., 2008). This could mean that differences in the presentation, development and progression of dementia in this group of people have been missed. It is these patients that currently pose the most challenges in terms of dementia diagnosis and therefore where research may have more benefit. This limitation is one that should be addressed in future research into this area. The studies that looked at this group in more detail found that symptomatology and carer reports were crucial to the diagnosis whereas cognitive assessment, although useful, was not it itself diagnostic (Deb, Hare \& Prior, 2007, Hithersay et al., 2019, Holland et al., 1998, Johansson, 2002).

There is currently no universally accepted cognitive assessment tool or standardised assessment routine for people with DS. This is reinforced by this review as each of the studies used a different set of cognitive assessments to measure change. Some of the assessments used were able to differentiate between baseline cognitive impairment and cognitive impairment associated with dementia. In particular delayed recognition and scrambled boxes showed a large effect size in relation to dementia diagnosis and a small effect size in relation to severity of ID, thus potentially differentiating between the two (Ball et al., 2008). Another study found 'reverse order' tests were sensitive to dementia but not mild/moderate level of intellectual disability (Johansson, 2002). The papers found in this review have utilised a variety of methods to study the development of dementia in people who have DS. Longitudinal study design allowed repeated assessment of cognition over time, either as part of the study or utilising previous assessments completed as part of routine care. Within these studies there was variation regarding how baseline severity of ID was assessed, including notes review, carer interview and assessment with cognitive batteries designed to estimate baseline ID (Hithersay et al., 2019, Krinsky-McHale et al., 2008, Margallo-Lana et al., 2007, McCarron et al., 2017, Oliver et al., 1998). These varied methods may have introduced bias to the studies especially when relying on carer memory. Other studies used a cross-sectional approach, again there was variety in how baseline severity of ID was assessed potentially significantly affecting the interpretation of decline and conclusions re how severity of ID impacts on dementia presentation and prognosis. Furthermore, these studies are assessing patients at one time point which is a limitation when investigating a condition that evolves over time (Ball et al., 2008, Holland et al., 1998, Johansson, 2002, Temple et al., 2001).

This review is limited by being unable to generate statistical comparison to allow more robust conclusions to be made. It examines $A D$ and dementia collectively to maximise inclusion of studies, however, people with different types of dementia experience cognitive decline at different rates and in different areas of cognition, potentially confounding the review findings. The review is limited by the studies included having multiple outcome measures of various validity and reliability and of which only some related to the research questions set out for this paper. In particular none of the papers found were specifically designed to answer the research questions posed by this review. The lack of papers available could represent a publication bias with papers finding a lack of association not being published, or it could represent that our question has not been addressed by current 
research. A further confounder is that concepts like cognitive reserve hypothesis which are being raised in Alzheimer's (Stern Y. 2012) has not been considered in the ID or DS populations.

\section{Conclusion}

Given the morbidity and mortality associated with dementia in people with DS it is important that there is a robust and accurate diagnostic pathway that is sensitive to capture the influences of ID severity and those of comorbid neuro-developmental/pervasive developmental disorders. There is the potential for standardised electronic patient/carer held records that will allow the integration of physician led assessment and patient/carer reports to provide an individualised holistic record of change.

There is limited evidence on how severity of ID impacts on the development, impact or resultant needs of dementia in people with DS particularly when the level of ID is more severe. There is no evidence regarding how comorbid neuro-developmental/pervasive developmental disorders may impact on the presentation, diagnosis, management or prognosis of dementia in people with DS. As a result of the lack of consistency between the studies that are available the results are difficult to compare and findings difficult to generalise into clinical practice.

This paper has identified multiple areas for future research including longitudinal studies into the presentation, development and progression of dementia in people with DS across all severities of ID, including people with comorbid pervasive developmental disorders. This is important to ensure people with DS receive parity of care, diagnosis and access to current and potential future treatments for dementia. 


\section{References}

Antonarakis, S. E., Lyle. R., Dermitzakis, E.T., Reymond, A., \& Deutsch, S. (2004). Chromosome 21 and down syndrome: from genomics to pathophysiology. Nature Reviews Genetics, 5, 725-738. doi: $10.1038 / \mathrm{nrg} 1448$

Ball, S. L., Holland, A. J., Treppner, P., Watson, P. C., \& Huppert, F. A. (2008). Executive dysfunction and its association with personality and behaviour changes in the development of Alzheimer's disease in adults with Down syndrome and mild to moderate learning disabilities. British Journal of Clinical Psychology, 47, 1-29. doi: 10.1348/014466507X230967

Biederman, J., Petty, C, R., Evans, M., Small J., \& Faraone, S, B. (2010) How persistent is ADHD? A controlled 10-year follow-up study of boys with ADHD. Psychiatry Research, 177, 299-304. doi: 10.1016/j.psychres.2009.12.010

The British Psychological Society (BPS). (2015). Dementia and People with Intellectual Disabilities: Guidance on the assessment, diagnosis, interventions and support of people with intellectual disabilities who develop dementia. Available at: https://www.dsrf.org/media/REP77\%20final\%20proof\%20(3).pdf [Accessed 25 ${ }^{\text {th }}$ April 2020]Braden, B .B., Smith, C. J., Thompson, A., Glaspy, T, K., Wood, E., Vatsa, D., ... Baxter, L. C. (2017) Executive Function and Functional and Structural Brain Differences in Middle-Age Adults With Autism Spectrum Disorder. Autism Research, 10, 1945-1959. doi: 10.1002/aur.1842|

Callahan, B. L., Bierstone, D., Stuss, D. T., Black, S. E. (2017) Adult ADHD: Risk Factor for Dementia or Phenotypic Mimic? Frontiers in Aging Neuroscience. 9, Article 260. doi: 10.3389/fnagi.2017.00260

Cosgrave, M.P., Tyrrell, J., McCarron, M., Gill, M., \& Lawlor, B.A. (2000) A five year follow-up study of dementia in persons with Down's Syndrome: early symptoms and patterns of deterioration. Irish Journal of Psychological Medicine, 17, 5-11. doi: 10.1017/S0790966700003943

Das, D., Cherbuin, N., Anstey, K, J., \& Easteal S. (2015). ADHD Symptoms and Cognitive Abilities in the Midlife Cohort of the PATH Through Life Study. Journal of Attention Disorders. 19, 414-24. doi: $10.1177 / 1087054712460887$

Deb, S., Hare, M., \& Prior, L. (2007) Symptoms of dementia among adults with Down's syndrome: a qualitative study. Journal of Intellectual Disability Research, 51, 726-739. doi: 10.1111/j.13652788.2007.00956.x

Ekstein, S., Glick, B., Weill, M., Kay, B., \& Berger, I. (2011). Down syndrome and attentiondeficit/hyperactivity disorder (ADHD). Journal of Child Neurology, 26, 1290-1295. doi: 10.1177/0883073811405201

Geurts, H. M., \& Vissers, M. E. (2012). Elderly with autism: Executive functions and memory. Journal of Autism and Developmental Disorders, 42, 665-675. doi: 10.1007/s10803-011-1291-0

Head, E., Doran, E., Nistor, M., Hill, M., Schmitt, F., Haier, R.J., \& Lott, I.T. (2011) Plasma A $\beta$ as a Function of Age, Level of Intellectual Disability and Presence of Dementia in Down syndrome. Journal of Alzheimer's Disease, 23, 399-409. doi: 10.3233/JAD-2010-101335 
Head, E., Powell, D., Gold, B, T., \& Schmitt, F, A. (2012) Alzheimer's Disease in Down Syndrome. European journal of neurodegenerative disease, 1, 353-364.

Hithersay, R., Startin, C.M., Hamburg, S., Mok, K.Y., Hardy, J., Fisher, E.M.C., Tybulewicz, V.L.J., Nizetic, D., \& Strydom, A. (2019) Association of Dementia With Mortality Among Adults With Down Syndrome Older Than 35 Years. JAMA Neurology, 76, 152-160. doi: 10.1001/jamaneurol.2018.3616

Holland, A.J., Hon, J., Huppert, F.A., Stevens, F., \& Watson, P. (1998) Population-based study of the prevalence and presentation of dementia in adults with Down's syndrome. British Journal of Psychiatry, 172, 493-498. doi: 10.1192/bjp.172.6.493

Hong, Q, N., Pluye, P., Fàbregues, S., Bartlett, G., Boardman, F., Cargo, M., Dagenais, P., Gagnon, MP., Griffiths, F., Nicolau, B., O'Cathain, A., Rousseau, M-C., \& Vedel, I. (2018) Mixed Methods Appraisal Tool (MMAT), version 2018. Registration of Copyright (\#1148552), Canadian Intellectual Property Office, Industry Canada.

Johansson, P.E. (2002) Development of an instrument for early detection of dementia in people with Down syndrome. Journal of Intellectual \& Developmental Disability. 27, 325-345. doi: $10.1080 / 1366825021000029357$

Kooij, S. J. J., Michielsen, M., Kruithof, H., \& Bijlenga, D. (2016). ADHD in old age: a review of the literature and proposal for assessment and treatment. Expert Review of Neurotherapeutics, 16, 1371-1381. doi: 10.1080/14737175.2016.1204914

Krinsky-McHale, S.J., Devenny, D.A., \& Silverman, W.P. (2002) Changes in explicit memory associated with early dementia in adults with Down's syndrome. Journal of Intellectual Disability Research, 46, 198-208. doi: 10.1046/j.1365-2788.2002.00365.x

Krinsky-McHale, S.J., Devenny, D.A., Kittler, P., \& Silverman, W. (2008) Selective attention deficits associated with mild cognitive impairment and early stage Alzheimer's disease in adults with Down syndrome. American Journal of Mental Retardation, 113, 369-86. doi: 10.1352/2008.113:369-386

Lejeune J, Gautier M, Turpin R. (1959). Etude des chromosomes somatiques de neuf enfants mongoliens. Comptes Rendus Hebdomadaires Des Seances, 248, 1721-1722.

Leonard, H., \& Wen, X. (2002). The epidemiology of mental retardation: challenges and opportunities in the new millennium. Mental Retardation and Developmental Disabilities Research Reviews, 8, 117-134. doi: 10.1002/mrdd.10031

Margallo-Lana, M. L., Moore, P. B., Kay, D. W. K., Perry, R. H., Reid, B. E., Berney, T. P., \& Tyrer, S. P. (2007). Fifteen-year follow-up of 92 hospitalized adults with Down's syndrome: incidence of cognitive decline, its relationship to age and neuropathology. Journal of Intellectual Disability Research, 51, 463-477. doi: 10.1111/j.1365-2788.2006.00902.x|

McCarron, M., McCallion, P., Reilly, E., \& Mulryan, N. (2014). A prospective 14-year longitudinal follow-up of dementia in persons with Down syndrome. Journal of Intellectual Disability Research, 58, 61-70. doi: 10.1111/jir.12074 
McCarron, M., McCallion, P., Reilly, E., Dunne, P., Carroll, R., Mulryan, N. (2017). A prospective 20year longitudinal follow-up of dementia in persons with Down syndrome. Journal of Intellectual Disability Research, 61, 843-852. doi: 10.1111/jir.12390

Morris JK, Springett A. (2014). The National Down Syndrome Cytogenetic Register for England and Wales 2013 Annual Report. Queen Mary University of London, Barts and The London School of Medicine and Dentistry.

Moss, J., Richards, C., Nelson, L., \& Oliver, C. (2013). Prevalence of autism spectrum disorder symptomatology and related behavioural characteristics in individuals with Down syndrome. Autism, 17, 390-404. doi: 10.1177/1362361312442790

National Institute of Child Health and Human Development (NICHD). (2017a). What are common symptoms of Down syndrome? Available at:

https://www.nichd.nih.gov/health/topics/down/conditioninfo/symptoms [Accessed 18th April 2020]

National Institute of Child Health and Human Development (NICHD). (2017b). What conditions or disorders are commonly associated with Down syndrome? Available at:

https://www.nichd.nih.gov/health/topics/down/conditioninfo/associated [Accessed 18th April 2020]

National Institute of Heath and Care Excellence (NICE). (2018a). Attention deficit hyperactivity disorder: diagnosis and management, NICE guideline [NG87]. Available at:

https://www.nice.org.uk/guidance/NG87 [Accessed 2nd April 2019].

National Institute of Heath and Care Excellence (NICE). (2018b). Dementia: assessment, management and support for people living with dementia and their carers, NICE guideline [NG97]. Available at: https://www.nice.org.uk/guidance/ng97/chapter/Recommendations [Accessed 22nd May 2019]

Oliver, C., Crayton, L., Holland, A., Hall, S., \& Bradbury, J. (1998) A four year prospective study of agerelated cognitive change in adults with Down's syndrome. Psychological Medicine, 28, 1365-1377. doi: 10.1017/S0033291798007417

Presson, A. P., Partyka, G., Jensen, K, M., Devine, O, J., Rasmussen, S, A., McCabe, L, L., \& McCabe, E. R. (2013). Current estimate of Down syndrome population prevalence in the United States. The Journal of Paediatrics, 163, 1163-1168. doi: 10.1016/j.jpeds.2013.06.013

Preferred Reporting Items for Systematic Reviews and Meta-Analyses (PRISMA). (2018). PRISMA for Scoping Reviews . Available at: http://www.prisma-statement.org/Extensions/ScopingReviews [Accessed 27th April 2020].

Richards, C., Jones, C., Groves, L., Moss, J., \& Oliver, C. (2015). Prevalence of autism spectrum disorder phenomenology in genetic disorders: a systematic review and meta-analysis. Lancet Psychiatry, 2, 909-916. doi: 10.1016/S2215-0366(15)00376-4

Sauna-Aho, O., Bjelogrlic-Laakso, N., Siren, A.,\& Arvio, M. (2018). Signs indicating dementia in Down, Williams and Fragile X syndromes. Molecular Genetics and Genomic Medicine, 6, 855-860. doi: 10.1002/mgg3.430 
Sinai, A., Mokrysz, C., Bernal, J., Bohnen, I., Bonell, S.,Courtenay, K., ... Strydom, A. (2018) Predictors of Age of Diagnosis and Survival of Alzheimer's Disease in Down Syndrome. Journal of Alzheimer's Disease, 61, 717-728. doi: 10.3233/JAD-170624

Stern Y. Cognitive reserve in ageing and Alzheimer's disease. Lancet Neurol. 2012;11(11):1006-1012. doi:10.1016/S1474-4422(12)70191-6

Strydom, A., Chan, T., King, M., Hassiotis, A., \& Livingston, G. (2013) Incidence of dementia in older adults with intellectual disabilities. Research in Developmental Disabilities, 34, 1881-1885. doi: 10.1016/j.ridd.2013.02.021

Temple, V., Jozsvai, E., Konstantareas, M.M., Hewitt, T.A. (2001) Alzheimer dementia in Down's syndrome: the relevance of cognitive ability. Journal of Intellectual Disability Research, 45, 47-55. doi: 10.1111/j.1365-2788.2001.00299.x

Walsh, D. M., Doran, E., Silverman, W., Tournay, A., Movsesyan, N., \& Lott, I. T. (2015). Rapid assessment of cognitive function in down syndrome across intellectual level and dementia status. Journal of Intellectual Disability Research, 59, 1071-1079. doi: 10.1111/jir.12200

Wiseman, F. K., Pulford, L. J., Barkus, C., Liao, F., Portelius, E., Webb, R., Chávez-Gutiérrez, L., Cleverley, K., Noy, S., Sheppard, O., Collins, T., Powell, C., Sarell, C. J., Rickman, M., Choong, X., Tosh, J, L., Siganporia, C., Whittaker, H, T., Stewart, F., Szaruga, M., Murphy, M, P., Blennow, K., de Strooper, B., Zetterberg, H., Bannerman, D., Holtzman, D, M., Tybulewicz, V, L, J., Fisher, E, M, C. (2018). Trisomy of human chromosome 21 enhances amyloid- $\beta$ deposition independently of an extra copy of APP. Brain, 141, 2457-2474. doi: 10.1093/brain/awy159

Yahia, S., El-Hadidy, M., El-Gilany, A., Amdel-Hady, D., Wahba, Y., \& Al-Haggar, M. (2014). Disruptive behavior in Down syndrome children: a cross-sectional comparative study. Annals of Saudi Medicine, 34, 517-521. doi: 10.5144/0256-4947.2014.517 


\section{Appendix 1 - Search terms}

\begin{tabular}{|c|c|}
\hline Search area & Search terms used \\
\hline \multirow{3}{*}{$\begin{array}{l}\text { Down } \\
\text { Syndrome }\end{array}$} & ("down* syndrome").ti,ab \\
\hline & ("trisomy 21").ti,ab \\
\hline & "DOWN SYNDROME"/ \\
\hline \multirow[t]{3}{*}{ Dementia } & (dementia).ti,ab \\
\hline & "ALZHEIMER DISEASE"/ OR DEMENTIA/ \\
\hline & (alzheimer*).ti,ab \\
\hline \multirow{10}{*}{$\begin{array}{l}\text { Pervasive } \\
\text { developmental } \\
\text { disorder }\end{array}$} & (autism OR autistic).ti,ab \\
\hline & (asperger*).ti,ab \\
\hline & (kanner OR kanners OR rett OR retts).ti,ab \\
\hline & ("pervasive development* disorder*").ti,ab \\
\hline & "LEARNING DISORDERS"/ \\
\hline & exp "CHILD DEVELOPMENT DISORDERS, PERVASIVE"/ \\
\hline & "RETT SYNDROME"/ \\
\hline & $\begin{array}{l}\text { ("non-verbal learning disorder*" OR "non-verbal learning disabilit*" OR "nonverbal } \\
\text { learning disorder*" OR "nonverbal learning disabilit*").ti,ab }\end{array}$ \\
\hline & (nonverbal OR non-verbal).ti,ab \\
\hline & (nld).ti,ab \\
\hline \multirow[t]{4}{*}{ ADHD } & ("attention deficit*" OR adhd).ti,ab \\
\hline & ("hyperactive disorder*" OR "hyperactivity disorder*").ti,ab \\
\hline & ("hyperkinetic disorder*" OR "hyper-kinetic disorder*").ti,ab \\
\hline & "ATTENTION DEFICIT DISORDER WITH HYPERACTIVITY"/ \\
\hline \multirow{8}{*}{$\begin{array}{l}\text { Intellectual } \\
\text { Disability }\end{array}$} & ("learning disabilit*").ti,ab \\
\hline & $\begin{array}{l}\text { ((mental* OR intellect* OR development*) ADJ1 (deficien* OR disabilit* OR } \\
\text { disabled OR retard* OR disorder* OR handicap* OR impair*)).ti,ab }\end{array}$ \\
\hline & ((intellect* OR mental) ADJ1 development*).ti,ab \\
\hline & ("cognitive retardation" OR "dull intelligence" OR "low intelligence").ti,ab \\
\hline & ("mental deficit").ti,ab \\
\hline & ("intellectual deficit").ti,ab \\
\hline & exp "INTELLECTUAL DISABILITY"/ \\
\hline & "COGNITIVE DYSFUNCTION"/ OR "intelligence quotient" OR cognit* OR iq \\
\hline \multirow{14}{*}{$\begin{array}{l}\text { Severity of } \\
\text { condition }\end{array}$} & (severity OR degree).ti,ab \\
\hline & (presentation).ti,ab \\
\hline & (comorbid*).ti,ab \\
\hline & (age ADJ1 diagnosis).ti,ab \\
\hline & (predict*).ti,ab \\
\hline & ("functional abilit*").ti,ab \\
\hline & (survival).ti,ab \\
\hline & "SURVIVAL RATE"/ \\
\hline & "AGE OF ONSET"/ \\
\hline & "RISK FACTORS"/ \\
\hline & ("risk factor*").ti,ab \\
\hline & (age ADJ1 onset).ti,ab \\
\hline & COMORBIDITY/ \\
\hline & "SEVERITY OF ILLNESS INDEX"/ \\
\hline
\end{tabular}


Pervasive Developmental Disorder search = 'Down Syndrome' AND 'Dementia' AND 'Pervasive Developmental Disorder'

ADHS search = 'Down Syndrome' AND 'Dementia' AND 'ADHD'

ID search = 'Down Syndrome' AND ‘Dementia' AND 'Intellectual Disability' AND 'Severity of condition' 